\title{
A case of dry beri-beri following bariatric surgery
}

\author{
Alice Verran¹, John Watkins ${ }^{1}$, Milan Piya1 ${ }^{1}$, Saboor Aftab1, Harpal S Randeva1, Sudhesh Kumar', \\ Sailesh Sankar ${ }^{2}$, Thomas M Barber ${ }^{1}$, Narendra L Reddy ${ }^{1}$ \\ Warwick Medical School' ${ }^{1}$, University of Warwick, Clinical Sciences Research Laboratories, Coventry, UK \\ University Hospitals of Coventry \& Warwickshire ${ }^{2}$, UK
}

\section{Introduction}

Over 9000 bariatric surgeries are conducted annually in the UK and post-operative micronutrient deficiency is common. We report a case of dry beriberi secondary to thiamine deficiency following Roux-en-Y bypass surgery.

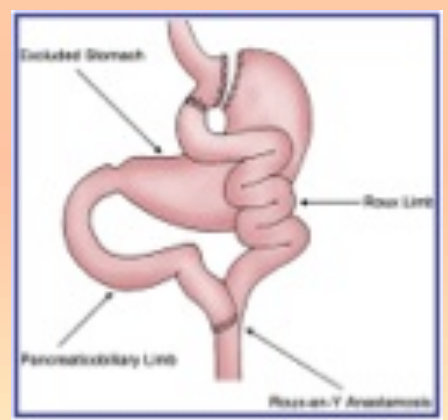

\section{Case history}

A 45-year old obese Caucasian lady (155 kg, BMI 57) presented with progressive proximal limb weakness, upper and lower limb paraesthesia, ataxia and athetoid tremors 18 months following a Roux-en-Y gastric bypass surgery. Postoperatively, she had lost $57 \mathrm{~kg}$ and maintained a healthy diet with moderate alcohol intake ( $<14$ units/week). She admitted non-compliance with multivitamin and Adcal D3 supplementation.

Examination revealed grade $3 / 5$ weakness in upper and lower limbs with impaired touch and vibration sensation in a 'glove and stocking' distribution. Deep tendon reflexes were reduced in lower limbs. Extrapyramidal and cerebellar functions were preserved.

\section{Management}

Nerve conduction studies revealed a mixed sensory and motor conduction defect. Serum calcium, vitamin B12, magnesium, ferritin, copper, phosphate and vitamin D levels were normal.

Initial thiamine status determination was unsuccessful due to unavailability of the test locally. Thiamine deficiency was diagnosed clinically and she was commenced on regular parenteral thiamine infusions $(100 \mathrm{mg} / \mathrm{day})$ and oral thiamine (300 mg/day). Serum thiamine after two thiamine infusions: $170 \mathrm{nmol} / \mathrm{L}(66-200)$.

Progress: Over next 24 months, complete resolution of proximal weakness and ataxia was noted. The tremors, mild paraesthesia and MRC grade 4 motor weakness of her hand muscles persist despite 3-monthly parenteral thiamine and vitamin B12 therapy, oral thiamine (300 mg/day), vitamin Bcompound and Adcal D3.

\section{Discussion}

Neurological complications after bariatric surgery are common, with peripheral neuropathies occurring in up to $16 \%$ patients(1).

Thiamine is alsorbed throughout the intestine and the mechanism by which it develops following gastric surgery is still being debated. Mechanisms suggested include prolonged nausea and vomiting and small bowel bacterial overgrowth(2).

Laboratory assessment of thiamine status is expensive, cumbersome and is not widely available. Samples must be frozen within 30 minutes of collection and exposure to light prevented. Whole blood thiamine testing is currently preferred but several alternative tests exist. Serum thiamine levels cannot be relied upon to exclude thiamine deficiency due to the low sensitivity and specificity of the test.
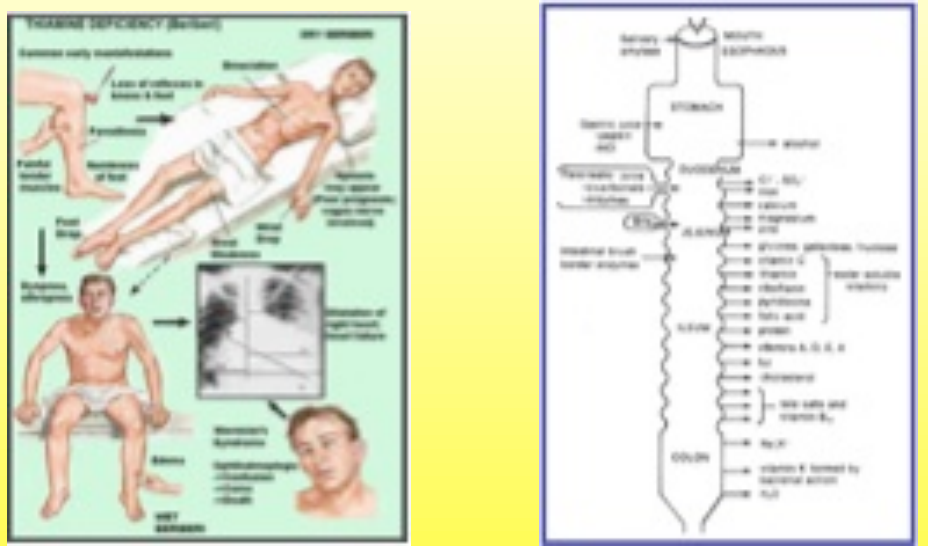

Symptoms of thiamine deficiency

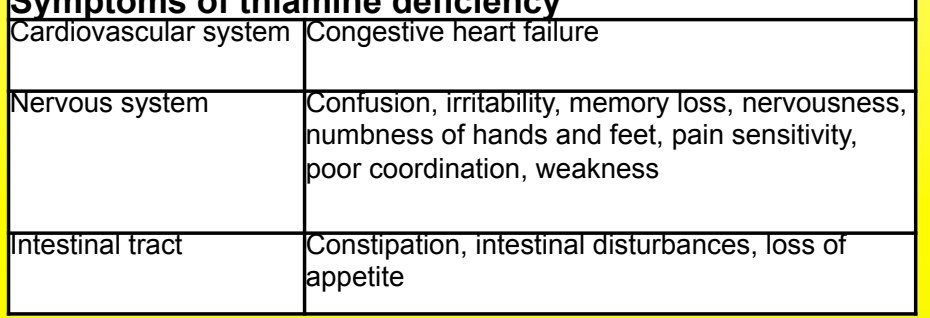

\section{Learning points}

A high index of clinical suspicion is therefore warranted to diagnose thiamine deficiency states (dry beriberi, wet beriberi and Wernicke Korsakoff syndrome).

- Biochemical and clinical monitoring of micronutrient deficiencies should be mandatory for all peri-operative bariatric surgery patients, preferably by adopting a locally agreed protocol. 